\title{
The processing of background noise in secondary path identification of Power transformer ANC system
}

\author{
YANG Peng ${ }^{a}$, YING Liming ${ }^{b}$, WANG Jinwei, WANG Guodong \\ School of Electrical Engineering, Wuhan University, Wuhan 430072, China \\ aemail:2014202070071@whu.edu.cn, bemail: Imying@whu.edu.cn
}

\begin{abstract}
Keywords: Power transformer Active noise control system; Secondary path; Identification; Background noise
\end{abstract}

\begin{abstract}
In secondary path identification of power transformer Active Noise Control (ANC) system, the background noise regards as useful signal, which makes identification result can't reflect the characteristic of secondary path and affect the stability and convergence of the ANC system. According to the actual working condition of Power transformer ANC system, the influence of background noise is almost removed by combining the time-sharing measurement method with signal correlation analysis and setting a threshold. The simulation results demonstrate that the accuracy of identification is significantly improved, showing the effectiveness of the proposed method.
\end{abstract}

\section{Introduction}

Active noise control (ANC) system of Power transformer based on Huygens' principle to achieve noise reduction. The secondary path transfer function applied to update the coefficient of adaptive control algorithm. Therefore, the performance of stability and convergence of the whole system are affected by the accuracy of identification. One of the key issue is how to improve the accuracy of identification.

There are two methods to identify the secondary path, offline identification and online identification. Off-line identification has several merits, such as fast convergent rate, easy to accomplishment and high accuracy of identification, etc. usually implemented in characteristic of secondary path changing slowly. In some practical cases, however, the secondary path can be time varying. It is necessary and practical to identify secondary path online. The injection of additional random white noise into the ANC system to estimate the secondary path in [1], which can adapt to the changes of the secondary path to provide the control system with more accurate secondary path parameters. However, the identification accuracy can be badly affected when the background noise is correlated with the useful signal. In order to solve this problem, the methods that can isolate the background noise to system signal have been proposed in [2]. The influence of background noise is completely removed, but there are many flaws in this method, such as structure complex, numerous calculations, narrowly application range. A number of algorithms without secondary path have been proposed in [3-5]. These algorithms optimize the objective function directly by avoided secondary path parameters. But the main problem is that the system unstable and difficult to convergence.

The influence of secondary path error for the system is discussed in this paper. The characteristic of background noise in substations is analyzed. According to the actual working condition in substations, the influence of background noise is almost removed by combined the time-sharing measurement method with signal correlation analysis and setting a threshold. The simulation results demonstrate that the accuracy of identification is significantly improved.

\section{Secondary path and its influence on control performance}

\section{The composition of the secondary path}

With the development of electronic components, Adaptive digital circuit replace the the analog circuit to be used in transformer ANC system. The block diagram of digital adaptive ANC system is 
shown in Fig. 1.

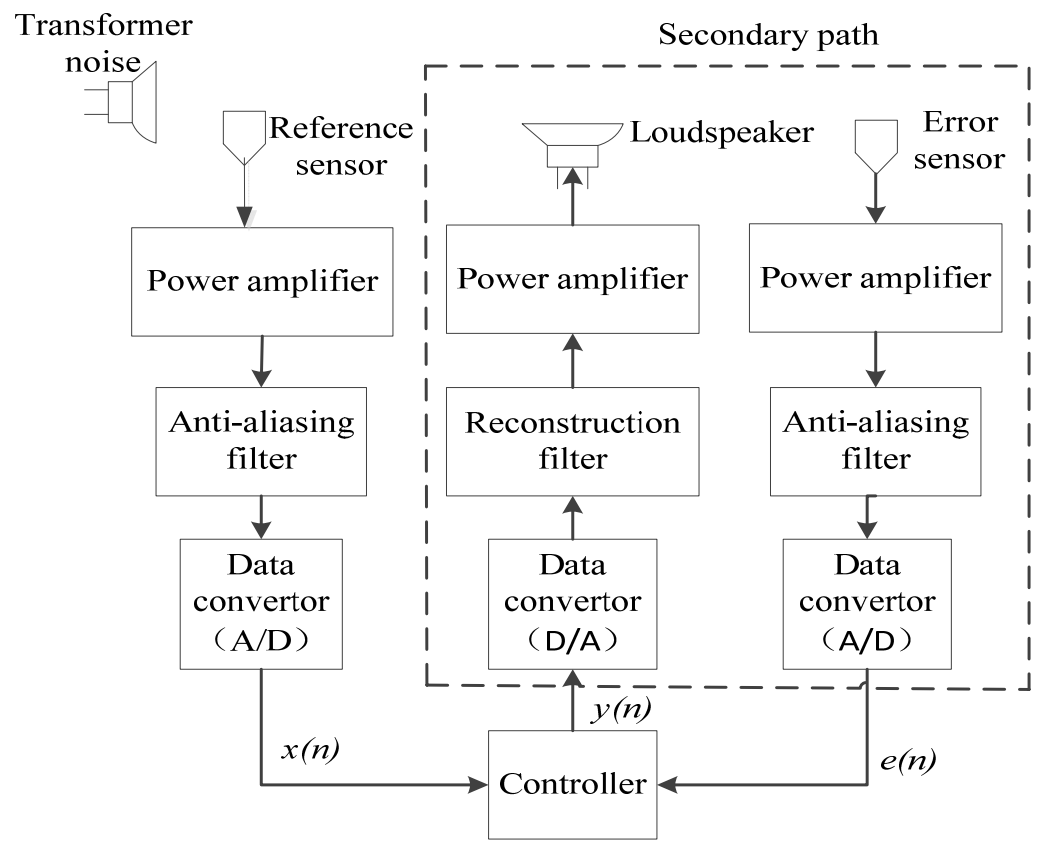

Fig. 1 The block diagram of ANC system

Where the reference $\operatorname{signal}_{x}(\mathrm{n})$ is fed through the controller to generate the signal $y(\mathrm{n})$ which drives the physical path, namely Secondary path, In the case of a digital active sound control system, the path consists of the loudspeakers with power amplifiers, physical space, microphones together with anti-aliasing filters and data convertors. The path outpute (n) is made up of the linear sum of the contributions from the secondary source and the effect of the primary source which is to be controlled. The presence of the physical path prevents the algorithm from being used to update weight coefficient of filter. Thus, we need to identify the transfer function of this physical path.

The error's impact on control system

The influence of identification error of secondary path on the stability and convergence of control algorithm have been analyzed in [6]. It was shown that the system, with a synchronously sampled sinusoidal reference signal of frequency $\mathrm{w}_{0}$, may be completely represented by an equivalent transfer function. The output of the adaptive filter $y$ (n) can be related to the error signal $e(\mathrm{n})$ by

$$
M(z)=\frac{Y(z)}{E(z)}=\beta\left[\frac{z \cos (\omega-\phi)-\cos \phi}{z^{2}-2 z \cos \omega_{0}+1}\right] .
$$

In which $\left|\hat{S}\left(\omega_{0}\right)\right|$ and $\phi$ are the amplitude and phase of $\hat{S}$, the estimate of the true transfer function $S$ at the frequency $\mathrm{w}_{0} \cdot \mu$ and $L$ denote Step size and the adaptive filter order separately.

Gives the important result

$$
\begin{aligned}
H(z) & =\frac{1}{1+S(z) M(z)} \\
& =\frac{z^{2}-2 z \cos \omega_{0}+1}{z^{2}-2 z \cos \omega_{0}+1+\beta S(z)\left[z \cos \left(\omega_{0}-\phi\right)-\cos \phi\right]}
\end{aligned}
$$

In the limit of very slow convergence $(\beta \rightarrow 0)$ where the dynamic properties of the error path become unimportant, and setting $S(z)=1$, then for a pure tone reference signal eqn. (2) becomes

$$
H(z)=\frac{z^{2}-2 z \cos \omega_{0}+1}{z^{2}-\left[2 \cos \omega_{0}-\beta \cos \left(\omega_{0}-\phi\right)\right] z+1-\beta \cos \phi} .
$$

This is a second-order recursive system whose stability can be investigated by examining 
whether the pole positions are within the unit circle. For small $\beta, H(z)$ will have conjugate poles at a distance of $r_{p}=\sqrt{1-\beta \cos \phi}$ from the origin. Since all the terms in $\beta$ are assumed positive, the distance of the poles from the unit circle can only be greater than one if $\cos \phi$ is negative, so the stability condition must be:

$$
\begin{aligned}
& r_{p}=\sqrt{1-\beta \cos \phi} . \\
& \cos \phi>0 \\
& -90^{\circ}<\phi<90^{\circ}
\end{aligned}
$$

The physical interpretation of this result is that, with a pure tone reference signal in the limit of slow adaption the estimate of the plant response need only have a phase response within $+90^{\circ}$ of the true phase response of the Secondary path for the system to converge as will as the reference signal is broadband noise signal.

Considering the convergence rate, the acceptable phase error range is smaller and to be inversely related to convergence step size. Take a system based on Fx-LMS algorithm for example:

The practical Fx-LMS algorithm is given by

$$
W(n+1)=W(n)-2 \mu e(n)[\hat{S}(z) X(n)] \cdot
$$

In the ideal situation, expression (7) can be written as

$$
W(n+1)=W(n)-2 \mu e(n) \hat{S}(\mathrm{z})[A+B \times W(n)] .
$$

Where, $A=E[d(\mathrm{n}) X(\mathrm{n})], B=E\left[X(\mathrm{n}) X^{T}(\mathrm{n})\right]$.

We define

$$
\begin{gathered}
\mathrm{V}(\mathrm{n})=W(n+1)-W(0) . \\
V^{\prime}(\mathrm{n})=Q^{-1} V(n)=Q^{T} V(n) .
\end{gathered}
$$

Where $Q$ is autocorrelation orthogonal Matrix, $Q^{-1}=Q^{T}$.The component $i$ makes the following relationship established.

$$
V_{i}^{\prime}(n+1)=V_{i}^{\prime}(n)\left[1-2 \mu \lambda_{i} \hat{S}(\mathrm{z})\right] \text {. }
$$

When $n \rightarrow \infty$, the convergence conditions of (11) must be:

$$
\left|1-2 \mu \hat{S}(\mathrm{z}) \lambda_{i}\right|<1 \text {. }
$$

Then, the convergence coefficient $\mu$ of Fx-LMS must be:

$$
0<\mu<\frac{\cos \phi}{\lambda_{i}|\hat{S}(z)|} \text {. }
$$

Where, $\phi$ is the phase error of the parameter identification in secondary path. $\lambda_{i}$ denote the eigenvalue of the Matrix. From (13), we can know that $\mu$ varies inversely to $|\hat{S}(z)|$ and is proportional to $\cos \phi$.It was proved that the phase error has little effect on $\mu$ when $\phi<45^{\circ}$, however, it can affect $\mu$ seriously when $\phi>70^{\circ}$, When the phase error is under $45^{\circ}$, it has little effect on $\mu$, but which more than 70 , seriously affect[6]. In this case, the systems make the control algorithm stable by reducing $\mu$, which will seriously reduce the rate of convergence.

\section{The processing of background noise}

In the process of parameter identification in secondary path, background noise is all kinds of acoustic signal such as transformer noise, corona discharge, ambient noise excepting for excitation signal used in recognition system. The noise usually breaks out abruptly and randomly, with its 
frequency band overlapped with excitation signal, and it can't be removed by Band-pass filter simply.

\section{The methods of removing background noise}

At present, two methods can remove the background noise, namely synchronization measurement method and Time-sharing measurement method.

(1) Synchronization measurement method, which is often used in many situations that background noise distributes uniformly, has two measurement channels. One is far away from the other and both are in the same direction. The signals, measured by the channel which is close to the sound source, are mixed by background noise and excitation signal, the other signal approximates to background noise. Two channels measurement at the same time can suppress the time error.

(2) Time-sharing measurement method, which is often used in many situations that background noise is time-invariant, requires making two signals measurements at two different time in the same place. Once to get the background noise signal before the system runs, and once to get the mixed signal after the system runs.

Taken together, Time-sharing measurement method can be used in secondary path parameter identification with easy operation and high precision, but there are issues to be resolved, such as the noise usually breaking out abruptly and randomly, with its frequency band overlapped with excitation signal.

\section{The characteristics and processing of different background noise}

(1) Random noise is characterised by high-amplitude and short-duration and can be removed if the amplitude is greater than our previously setting threshold which is the average of several sampling periods.

(2) When the frequency band of background noise overlapped with excitation signal, we must make a correlation analysis on them. Cross-Correlation Function of two signals, $x(t)$ and $y(t)$, can be defined as

$$
R_{x y}(\tau)=\frac{1}{T} \int_{0}^{T} x(t) y(t+\tau) d t .
$$

Where, $T$ is the correlation time. $\tau$ denotes the interval time between two signals. When the frequency of the two signals are $\omega$, namely $x(t)=a \sin (\omega t+\varphi)$ and $y(t)=b \sin (\omega t+\varphi-\theta)$. We can written expression (14) as

$$
\begin{aligned}
R_{x y} & =\frac{1}{T_{0}} \int_{0}^{T_{0}} a b \sin \left(\omega t+\varphi_{L}\right) \sin \left(\omega(t+\tau)+\varphi_{2}-\theta\right) d t \\
& =\frac{1}{2} a b \cos (\omega \tau-\theta)
\end{aligned}
$$

From expression (15) we can know that if two signals have the same frequency, the correlation function will retain the amplitude and phase of the original signal. Otherwise, the correlation function will become zero.

When two signals are mixed-signals with different frequencies, namely $x(t)=\sum_{i=1}^{m} a_{i} \sin \left(\omega_{i} t+\varphi\right)$ and $y(t)=\sum_{j=1}^{n} b_{j} \sin \left(\omega_{j} t+\varphi-\theta_{j}\right)$, Cross-Correlation Function can be written as

$$
\begin{aligned}
R_{x y} & =\frac{1}{T_{0}} \int_{0}^{T_{0}}\left[\sum_{i=0}^{m} a_{i} \sin \left(\omega_{i} t+\varphi\right)\right]\left[\sum_{j=0}^{n} b_{j} \sin \left(\omega_{j} t+\varphi-\theta_{j}\right)\right] d t \\
& =\frac{1}{2} \sum_{s=0}^{k} a_{s} b_{s} \cos \left(\omega_{s} \tau-\theta_{s}\right)
\end{aligned} .
$$

Where, $\mathrm{m}$ and $\mathrm{n}$ is the frequency numbers of the two mixed signals. $K$ is the numbers of signal 
which have the same frequency, such as $\omega_{s}$, in the mixed signal. $a_{s}$ and $b_{s}$ denotes the amplitude . The correlation function will retain the amplitude and phase of the original signals which have same frequency. The correlation functions are processed by a Fourier transformation so that the same frequency band can be extracted.

So the influence of background noise in substations can be completely removed by combining the time-sharing measurement method with signal correlation analysis.

\section{Simulation}

Off-line identification has several merits, such as fast convergent rate, good operation stability and high identification accuracy. So it is suitable for theoretical and experimental research. To study more realistic situations, we use noise signal of $110 \mathrm{KV}$ transformer substation as the background noise. First, frequency spectrum analysis on the noise, from the results shown in Fig.2, it can be seen that the frequency of the noise are mainly distributing on $100 \mathrm{~Hz}, 200 \mathrm{~Hz}, 300 \mathrm{~Hz}$, $400 \mathrm{~Hz}$ and $500 \mathrm{~Hz}$. The excitation signal of recognition system is sinusoidal signal, the amplitude has same magnitude with the noise and the frequency is $1400 \mathrm{~Hz}$. convergence step is $\mathrm{u}=10$, Sampling Frequency is $\mathrm{fs}=3000 \mathrm{~Hz}$. First, there is no processing of the background noise. From Fig.3 we can see that the error signal have a tendency to converge to zero, but fluctuated greatly, and the output signal was distorted. The reason is that the background noise regards as useful signal, which makes identification result can't reflect the characteristic of secondary path. So we remove the influence of the background noise in off-line identification by the proposed method. The parameters used in the simulation are same with before. The results are showing flown.

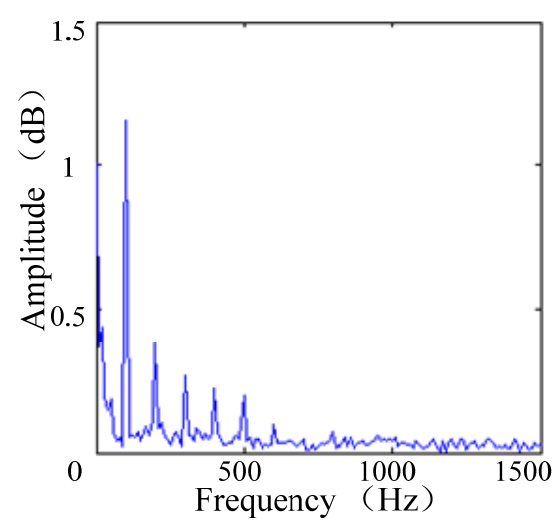

Fig.2 The spectrum of transformer noise
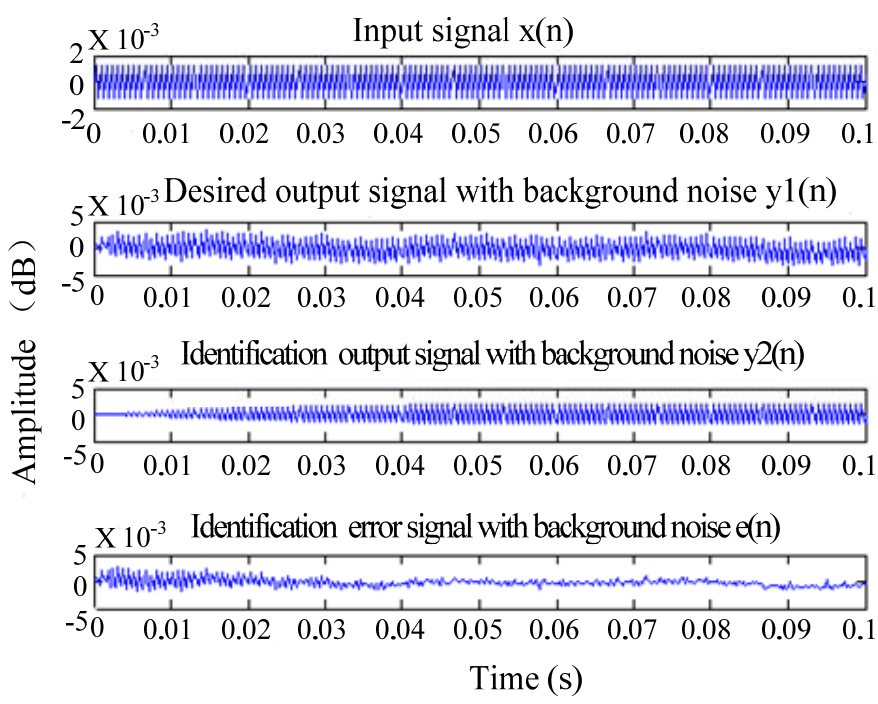

Fig.3 The simulation results with background noise

Fig.4 show the spectrum of mixed signals includes excitation signal which frequency is $1400 \mathrm{~Hz}$ and other background noise's frequency are mainly distributing on $100 \mathrm{~Hz}, 200 \mathrm{~Hz}, 300 \mathrm{~Hz}$, $400 \mathrm{~Hz}$ and $500 \mathrm{~Hz}$. Background noise has been removed and the restored output is close to sinusoidal signal after signal correlation analysis show in Fig.4 and Fig.5. From Fig.5 and Fig.6, we can know that the identification error is converged at 0.06 seconds and the frequency characteristics of the identification transfer function are consistent with the settings. In conclusion, the estimation errors have good steady-state performance and converge very fast after the background noise has been removed. 

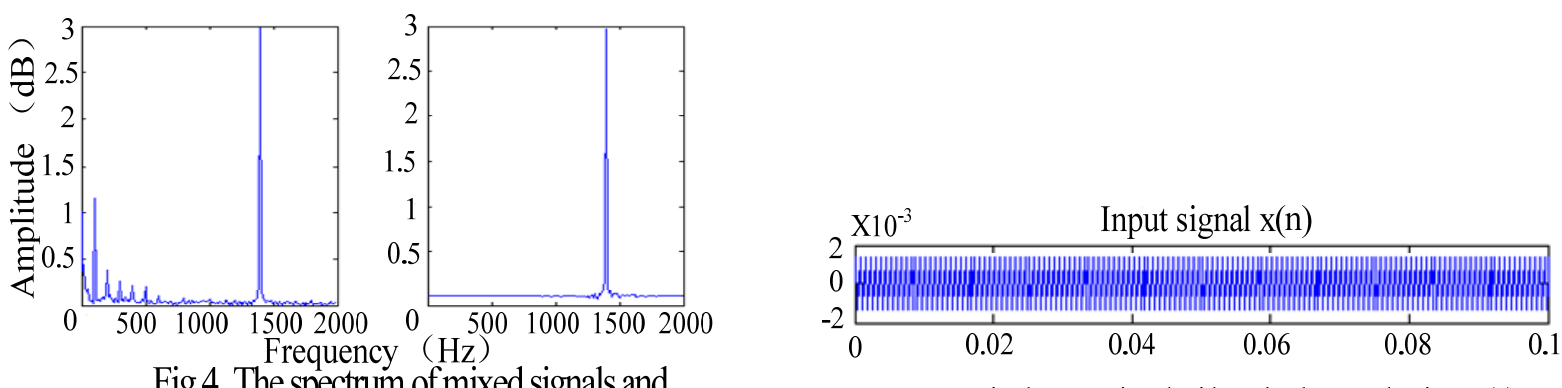

Fig.4 The spectrum of mixed signals and

After removing the background noise

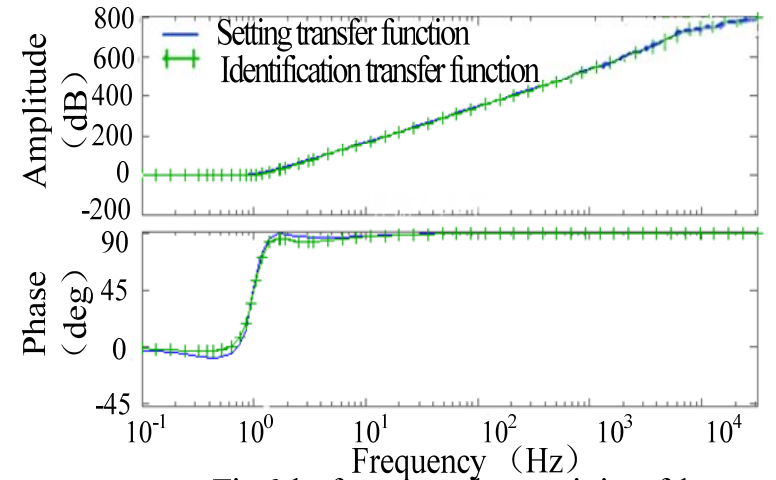

Fig.6 the frequency characteristics of the identification transfer function and the settings
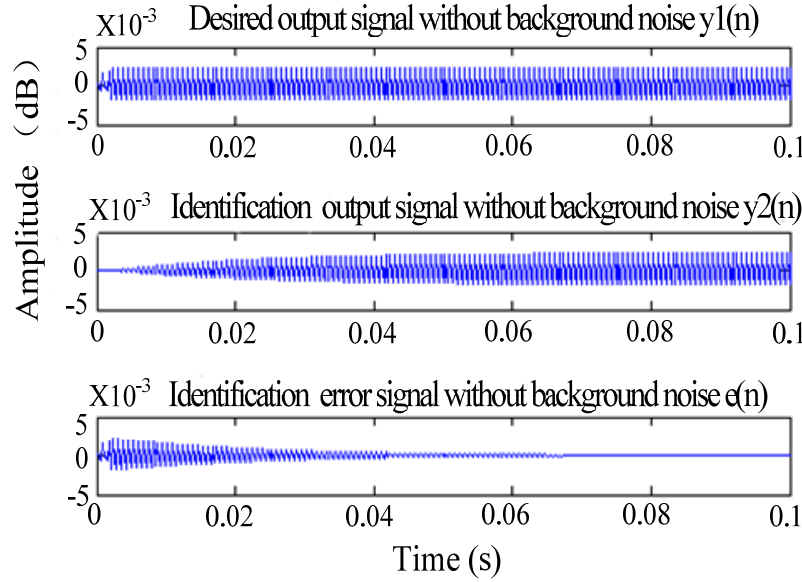

Fig.5 The simulation results without background noise

\section{Conclusion}

The estimation errors have good steady-state performance and converge very fast after the background noise has been removed by combining the time-sharing measurement method with signal correlation analysis and setting a threshold. The simulation results demonstrate that the accuracy of identification is significantly improved, showing the effectiveness of the proposed method.

\section{Acknowledgments}

Project supported by the National Natural Science Foundation of China (No. 51377123).

\section{References}

[1] LJ Eriksson, MC Allie. Use of random noise for on-line transducer modeling in an adaptive active attenuation system [J].The Journal of the Acoustical Society of America, 1989, 85(2):797-802.

[2] Zhang M, Lan H, Ser W. Cross-updated active noise control system with online secondary path modeling [J]. IEEE Transactions on Speech and Audio Processing, 2001, 9(5): 598-602.

[3] D. Zhou, V. De Brunner. A New Active Noise Control Algorithm That Requires No Secondary Path Identification Based on the SPR Property [J]. IEEE Trans. Signal Process, 2007, 55(5): 1719-1729.

[4] E. Bjarnason. Algorithms for Active Noise Cancellation without Exact Knowledge of the Error-path Filter [C]. IEEE Int. Symp. On Circuits and Systems. 1994: 573-576.

[5] Cheng-Yuan Chang, Deng-Rui Chen, "Active Noise Cancellation without Secondary Path Identification by Using an Adaptive Genetic Algorithm." IEEE Trans. On Institution and Measurement, 2010, 59(9): 2315-2327. 
[6] C. C. Boucher, S. J. Elliott, P. A. Nelson. Effect of errors in the plant model on the Performance of algorithms for adaptive feed-forward control, proceedings of IEEE, 1991, 138(4), 313-319.

[7] K. Fujii, J. Ohga. Method to update the coefficients of the secondary path filter under active noise control. Signal Processing, 2001, 81, 381-387.

[8] V. DeBrunner, L. DeBrunner, and L. Wang, "Subband adaptive filtering with delay compensation for active control," IEEE Trans. Signal Process" vol. 52, no. 10, pp. 2932-2941, Oct. 2004,

[9] C. Y. Chang and K. K. Shyu, "Active noise cancellation with a fuzzy adaptive filtered-X algorithm,” Proc. Inst. Elect. Eng.-Circuits Devices Syst., vol. 150, no. 5, pp. 416-422, Oct. 2003.

[10]Y. Tan, Y. He, C. Cui, and G. Qiu, "A novel method for analog fault diagnosis based on neural networks and genetic algorithms," IEEE Trans. Instrum. Meas., vol. 57, no. 11, pp. 2631-2639, Nov. 2008.

[11]H. Lan, M. Zhang, and W. Ser, "An active noise control system using online secondary path modeling with reduced auxiliary noise," IEEE Signal Process. Lett, vol. 9, pp. 16-18, Jan. 2002. 